\title{
History of High-Resolution Anoscopy
}

\author{
S. David Cho, MD ${ }^{1}$ Emily Groves, MD $^{2}$ Victoria V. Lao, MD ${ }^{3}$
}

1 Department of Surgery, Kaiser Permanente, Portland, Oregon

2 Department of General Surgery, Oregon Health and Science University, Portland, Oregon

${ }^{3}$ Department of General Surgery, University of Washington,

Seattle, Washington

Address for correspondence S. David Cho, MD, Department of Surgery, Kaiser Permanente, 9900 SE Sunnyside Road, Clackamas, OR 97015 (e-mail: sungeyun@hotmail.com).

Clin Colon Rectal Surg 2018;31:336-346.

\begin{abstract}
Keywords

- high-resolution anoscopy

- anal dysplasia

- anal cancer

- anal intraepithelial lesions

High-resolution anoscopy (HRA) is a form of low-resolution anal microscopy currently utilized in the screening and management of anal squamous dysplasia. No randomized controlled trials, national or international guidelines exist on the use of HRA for this purpose. Much of our understanding of this entity has been adapted from the literature on cervical squamous dysplasia, including the technique of HRA itself. Epidemiologic evidence has shown that the prevalence and incidence of anal dysplasia is highest in HIV-positive populations. The history of this technique parallels the evolution of our understanding of anal dysplasia. To understand the history of the use of HRA and its place in the screening and management of anal squamous dysplasia, we discuss key advances in the understanding of human papillomavirus-related squamous dysplasia. We begin with early reports in the field establishing the link between this virus and squamous dysplasia, through the marked increase in anal cancer seen with the onset of the HIV epidemic, the identification of relevant populations at risk, the performance of the test itself, to its use today.
\end{abstract}

The history of high-resolution anoscopy (HRA) in the management of anal squamous dysplasia or squamous intraepithelial lesions (SIL) in many ways parallels the evolution of our understanding of this entity and of this field itself. Exploring the history of this technique is to examine how several important questions came to be answered over time-"What is the technique?" "Why did we start using this technique?" "Who is this technique effective for?" "How do we know it is effective?"

It is commonly inferred that HRA and its uses were adapted from the management of cervical squamous lesions. It is important in this discussion to understand the historical context to explore the evolution and use of colposcopy, the similarities between cervical dysplasia and anal squamous dysplasia, and the role of human papillomavirus (HPV) in these two diseases. As such, the answers to the above questions can be framed by a series of statements that outline major steps-from the early days in the field to today-that shaped our current usage of this technique. - Table 1 outlines this progression.

Issue Theme Anal Intraepithelial Neoplasia and Squamous Cell Cancer of the Anus; Guest Editor: Fia Yi, MD, FACS, FASCRS
Internationally recognized guidelines based on high-quality evidence for the use of HRA do not exist. There are no randomized data that have been used to formulate guidelines or institute treatment guidelines. Therefore, an understanding of the inferential steps that have been made over time can help us understand where we are today. A comprehensive discussion of the entire field is outside the scope of this article. Our intent is not to summarize the entirety of the field, but to briefly discuss notable progressions in understanding of pathophysiology and management of SIL, define high-risk populations who we currently understand benefit most from HRA, and review current utilization practices.

\section{Human Papillomavirus Leads to Cervical Dysplasia and Cervical Dysplasia Leads to Cervical Cancer}

Much of our current understanding of anal cancer has been derived from the body of literature describing cervical cancer owing to the shared similarities between the two pathologic processes.
Copyright (c) 2018 by Thieme Medical Publishers, Inc., 333 Seventh Avenue, New York, NY 10001, USA. Tel: +1(212) 584-4662. ISSN 1531-0043. 
Table 1

\begin{tabular}{|l|}
\hline $\begin{array}{l}\text { I. Cervical dysplasia is caused by HPV and cervical } \\
\text { dysplasia leads to cervical cancer. }\end{array}$ \\
\hline $\begin{array}{l}\text { II. Colposcopy can be used to detect cervical } \\
\text { squamous lesions. }\end{array}$ \\
\hline $\begin{array}{l}\text { III. Colposcopy today has a defined role in screening } \\
\text { for cervical SIL. }\end{array}$ \\
\hline IV. Colposcopy can be similarly used for anorectal disease. \\
\hline V. HRA can be used to detect anal dysplasia. \\
\hline $\begin{array}{l}\text { VI. HPV similarly causes anal dysplasia and anal } \\
\text { dysplasia can cause anal cancer. }\end{array}$ \\
\hline $\begin{array}{l}\text { VII. Those at highest risk for anal cancer were identified } \\
\text { as MSM who are HIV positive (HIV }+ \text { ). }\end{array}$ \\
\hline $\begin{array}{l}\text { VIII. HRA is therefore used most often to detect SIL in } \\
\text { HIV }+ \text { MSM. }\end{array}$ \\
\hline $\begin{array}{l}\text { IX. How is HRA used now and how well does HRA work } \\
\text { to detect SIL in HIV }+ \text { MSM? }\end{array}$ \\
\hline X. How will HRA be used in the future? \\
\hline
\end{tabular}

Abbreviations: HPV, human papillomavirus; HRA, high-resolution anoscopy; MSM, men who practice anoreceptive intercourse with men; SIL, squamous intraepithelial lesion.

By the early 1970s, it was proposed that cervical cancer was associated with several factors including early coitus, multiple partners, and low socioeconomic status; so, it was surmised that some sort of venereal disease was involved or at least implicated in the development of cervical cancer. ${ }^{1}$ Condyloma had been recognized for some time, but not studied extensively at the molecular level until the 1960s, when use of the electron microscope became more commonplace. The presence of viral particles, known as "human papova virus" had already been established in skin warts ${ }^{2}$ when Almeida and colleagues set out to study condyloma. ${ }^{3}$ They isolated a similar virus in 13 of 25 warts examined by electron microscopy. As colposcopy gained ground, smaller and smaller lesions of various etiologies were being found and studied. No longer were detectable cervical or vulvar lesions palpable, or even macroscopically visible but subclinical pathology could be observed at very early stages. Case reports ${ }^{4,5}$ were published about an entity that came to be known as "noncondylomatous cervical wart infection." These lesions were initially thought to be due to a different virus than the one isolated in condyloma due to the different macroscopic appearance of the lesion. However, Reid and colleagues ${ }^{6}$ established the connection between HPV and these "noncondylomatous warts" when papova viral particles were isolated from nearly half of cervical cytologic smear specimens from patients with the disease. They concluded that genital HPV infection can produce overt condylomas or what they termed subclinical "noncondylomatous warts." The term "noncondylomatous warts" was replaced with subclinical papillomavirus infection (SPI). By the early 1980 s, defining this entity allowed the group to study this population over time and establish the diagnosis as it related to cervical cancer.
Toward the end of understanding SPI, Reid et al undertook a cross-sectional study of 160 women. Forty of these had undergone radical hysterectomy for stage IA or IB cervical cancer and 40 had cervical intraepithelial neoplasia (CIN) I or III. The surgical indication for these women was premalignancy in the group with CIN III, and the CIN I group comprised of women who underwent hysterectomy for benign conditions and were found to have CIN I on pathology review. These 80 women were the study group. Eighty matched control patients were found without CIN or cervical cancer. They found HPV in $91 \%$ of CIN/ cervical cancer patients and only $12 \%$ of controls, raising the possibility of a causal relationship between the virus and cancer. ${ }^{7}$ Subsequently, Reid ${ }^{8,9}$ explored this concept further by examining 52 women using colposcopy, acquiring 72 biopsies for morphologic characteristics associated with SPI and CIN I-III. He discovered that on histologic examination, SPI is not a different entity from CIN, but the early end of a spectrum including all of these entities. That is, the further along the spectrum between SPI and CIN III cells progress, the more atypia they show. Since HPV was implicated in SPI and SPI was found to be an early manifestation as the same entity as CIN, HPV was established as a causal agent for cervical dysplasia, the precursor to cervical cancer.

Around the same time, Pilotti and colleagues ${ }^{10}$ sought also to clarify the relationship of HPV and CIN. They looked at 100 cervical cone specimens of advanced CIN, and found that 56 of these were associated with viral cytopathic effects in DNA attributable to the HPV genome, most of which were associated with "flat condyloma." The cytologic sensitivity of finding the changes associated with HPV was 95\% in the CINII group, and $81 \%$ in the CIN III group.

Once the link between HPV and cervical dysplasia was established, other investigators built upon this foundation and strengthened the link between cervical dysplasia and cervical carcinoma. Boshart and coworkers ${ }^{11}$ made a breakthrough in 1984 by isolating HPV subtype 18 from cervical cancer specimens. They noted that compared with other HPV subtypes, types 16 and 18 "reveal some remarkable features: they exhibit a surprisingly specific association with malignant genital tumors. HPV 18 thus far has been exclusively detected within malignant tissue, and HPV 16 is also regularly present in premalignant 'high-risk' lesions."

Ho and colleagues ${ }^{12}$ took a population-based approach to this question by following up 608 college women over an average of 2.2 years via nearly 3,000 visits. They found that the cumulative incidence of HPV over 3 years was found to be $43 \%$ in sexually active college women. Furthermore, most HPV infections were transient, dropping to $9 \%$ over 3 years. However, HPV subtypes 16 and 18 were among the most persistent over the study period and in fact associated with the longest duration of infection. The incidence of SIL was $31 \%$. Among those with SIL, its development was associated with persistent infection with high-risk subtypes.

The above evidence provided the link between SIL and an infectious etiology causing changes at the cytologic level which could be seen earlier than lesions visible to the naked eye. In 1984, Reid et $\mathrm{al}^{9}$ noted that "CIN represents a single population with a pyramidal distribution, in which most 
cases do not progress from one level of severity to the next." The characteristics of high prevalence, link to carcinoma, and clinically feasible detectability led early investigators to the development of tools to detect early cervical lesions.

\section{Colposcopy Can Be Used to Detect Cervical Squamous Lesions}

The advent of colposcopy can be traced to Germany in the early 1920 s and is the subject of a fascinating, if poorly translated, report by Fusco et $\mathrm{al}^{13}$ in 2008 . Hans Hinselmann first published on colposcopy in 1925 in an effort to evaluate cervical lesions in vivo. Early iterations of the examination involved externalizing the cervix and inspection under a microscope. At that time, the diagnosis of cervical cancer was considered early if the mass was “the size of a pigeon's egg." In 1925, Austrian histologist Walter Schiller observed that dysplastic and neoplastic tissues do not take up glycogen. He therefore proposed that staining tissues with an iodine solution would highlight tissues that did not contain iodine-binding glycogen. He selected an iodine solution developed by French physician Jean Guillaume Auguste Lugol. By 1938, Hinselmann incorporated both Lugol's iodine and acetic acid into the colposcopy procedure, improving visibility. With this method, he could detect much smaller foci of cervical cancer. Most importantly, he began to emphasize and publish on epithelial and histologic changes rather than grossly macroscopic. It took until 1949 to devise a microscope with direct illumination that was able to evaluate epithelial changes after application of topical solutions, in effect the first colposcope containing the elements known today. As the method was challenging to learn, not all surgeons had the appropriate equipment available, and due in part to Hinselmann's activity in the Nazi party, it took until the mid-1950s to 1960 s for colposcopy to become adapted worldwide.

Dr. George Papanicoloau first began work on cervicovaginal cytology in the 1920s and published his work in $1941 .{ }^{14}$ Although no large randomized controlled trials have been conducted, epidemiologic evidence worldwide has demonstrated the effectiveness of his technique. In the United States, rates of cervical cancer decreased from 36.3 per 100,000 women in the 1930 s to 7.2 per 100,000 women in the 1990s. ${ }^{14}$ Cytologic screening has since gained popularity in the United States, but at that time the only intervention available was conization to evaluate histology. This was rather invasive for low-grade dysplasia, and further complicating the issue was the low sensitivity of traditional cervical cytology (51\%). ${ }^{14}$ Therefore, many gynecologists clinically followed abnormal cytology until pathology became apparent. Thus arose the need for a method of closely monitoring the cervix, and development of less invasive tissue collection techniques for diagnosis and intervention. This is one of the reasons that colposcopy gained ground in the 1960s and 1970s-initially as an efficient and effective means of following patients with abnormal cytology clinically. ${ }^{15}$ One other proposed reason for the increased utilization of colposcopic technique around this time was the markedly increased incidence of HPV thought to be due to societal behavior changes in the 1960 s. $^{16}$
By the mid-1970s, colposcopy was becoming the standard follow-up method for women with cervical intraepithelial neoplasia detected on Pap smear cytology. This regimen brought about increased detection rates of asymptomatic cervical metaplasia. Krumholz and Knapp ${ }^{17}$ showed that colposcopically directed biopsies achieved a correct diagnosis in nearly $90 \%$ of women compared with $50 \%$ by random biopsy in those who had abnormal pap smears. The results were compared with cervical conization, the gold standard at the time. This obviated the need for conization in the majority of patients.

As collective colposcopic skills improved, so did descriptions of colposcopic findings. These descriptions of pathology become more uniform over the years. A common vernacular surrounding skin lesion appearance and correlation to histologic grade began to develop. ${ }^{9,16}$ Sub-characteristics of acetowhite lesions were described, refined, and codified. Changes to both the epithelium and associated vasculature were noted. Findings such as leukoplakia, mosaicism, micropapillary projections ("Brain-like" in appearance), and sharpness of the margin of the lesion were described and assigned varying degrees of significance. Importantly, these observations were found to correlate histologically with premalignant lesions. ${ }^{16}$

Colposcopy became used more frequently to assess noncervical disease. In a report by Burke et $\mathrm{al}^{18}$ in 1974, colposcopy was used to assess the vaginal wall. Distinct findings consistent with vaginal adenosis, found in female offspring of mothers who took stilbestrol during pregnancy, accurately correlated with malignant and premalignant vaginal lesions. The colposcopic findings were similar to the lesions described in the cervix, including mosaicism patterns and acetowhite epithelium. The consistency of these findings led to colposcopy being used to survey vaginal lesions in addition to cervical lesions. This provided further strength to the consistency and correlation between colposcopic findings and premalignancy.

\section{Colposcopy Today Has a Defined Role in Screening for Cervical SIL}

The body of data and the historical review provided have laid the framework of how colposcopy has come to be used today. Interventions developed specifically for this method further solidified its usage. As an example, in the 1980s laser procedures were developed for dysplasia diagnosed by colposcopy. This practice evolved into the loop electrical excision procedure (LEEP), whereby a small segment of tissue is excised using a wire loop to which current is applied. ${ }^{15}$

Current screening practices include cytology starting at age 21. ${ }^{19}$ This is done in conjunction with HPV co-testing, and HPV is sent for sequencing to determine type. Any dysplasia or positive HPV test is then followed by colposcopy with endocervical sampling. Since cytology is an important determinant of who undergoes colposcopy, a workshop under the auspices of the National Cancer Institute was convened in 1988 to help standardize the reporting of cervical cytologic screening. This group introduced the term squamous intraepithelial lesion or SIL. ${ }^{20}$ Subsequent refinements to terminology are based on this original publication. While the sensitivity of cytology is low, and the false negative rate high, co-testing of cytology with HPV test has increased sensitivity. ${ }^{14,19}$ Screening has 
shown to be effective. Mortality from cervical cancer has improved from $5.55 / 100,000$ in 1975 to 2.38 in the late 2000 s. $^{21}$ Cervical cancer is found in $2 \%$ of women with highgrade SIL (HSIL) on cytology. ${ }^{19}$ In general, and as noted above, abnormal cytology with high-risk HPV co-testing or known HPV positivity prompts colposcopy. Its role is well defined based on characteristics such as age, HPV subtype, cytologic findings, increase or decrease in atypia, and special populations such as immunocompromised, pregnancy, very young age, and postmenopause. ${ }^{19,21,22}$

\section{Colposcopy Can Be Similarly Used for Anorectal Disease}

Reports like those of Burke et $\mathrm{l}^{18}$ provided a logical foundation to apply the colposcopic technique to other areas bearing squamous epithelium like the anus. Colposcopy's success as a minimally invasive technique detecting subclinical premalignant precursors made it attractive. In summarizing the parallels and logical transition from colposcopic survey of the cervix to that of the anal canal, Darragh and Winkler ${ }^{23}$ aptly stated:

"The success of cervical cancer screening has led to its use as a template for anal cancer screening in high-risk groups.... Parallels between the models for anal cancer screening and cervical cancer screening are also based on the biologic and morphologic similarities between anal intraepithelial neoplasia (AIN) and CIN. ... On the clinical side, there are also many similarities between anal and cervical HPV-related squamous mucosal disease. ... Clinical approaches to the diagnosis of AIN also borrow from the cervical cancer model and include the application of colposcopy to the evaluation of the anal canal and perianal region. This has led to the development of a new subspecialty of colposcopy referred to as high resolution anoscopy (HRA)."

\section{What Is HRA?}

Colposcopy of the uterine cervix and HRA are considered equivalent terms for the same procedure with the same intent in each respective anatomic location. HRA is a visual inspection of the transition zone, anal canal, and perianal skin under magnification. Along with the application of acetic acid and Lugol's iodine, the goal is to render visible and prominent certain characteristic .changes representative of underlying dysplasia. The technique is briefly described here. ${ }^{24,25}$

The device is a low-power, stereoscopic field microscope. The scope head or "optics carrier" consists of the objective lens, connected to a light source and binocular eyepieces or video output to a screen. Often there are several lenses to allow for various amounts of magnification (typically between $8 \times$ and $20 \times$ ) and variable working distance. Lower magnification yields a wider view and greater depth of field while more magnification reveals finer features. A focal distance of approximately 250 to $300 \mathrm{~mm}$ allows for the anoscopist to be close enough to obtain biopsies, but far enough to utilize the magnification provided. There are also green and blue filters which can improve assessment of acetowhitening as well as the appearance of blood vessels. Similar to conventional microscopy, adjustments to the light source and focus can be made. The whole apparatus is mounted on a rolling pedestal and has a rotating head to allow for varied heights and positioning of the optics carrier.

The procedure itself can be completed in $\sim 10$ minutes. Maximal patient comfort is paramount. Typically, a left or right lateral position is undertaken, pulling the knees in toward the chest. A lubricated anoscope is directed into the anus. A long cotton swab wrapped with gauze soaked in a $3 \%$ acetate solution is then inserted through the anoscope, and the anoscope is withdrawn with the gauze retained in the anal canal. After 2 to 5 minutes, the gauze is removed. Dysplastic tissue will turn white after contact with the acetic acid (acetowhitening). The anoscope is reinserted and the anal canal, transition zone, and perianal skin are carefully examined. Staining the tissue with an iodinebased Lugol's solution is also commonly used. The degree of dysplasia (AIN I, II, III) can be determined visually and biopsies are then taken of suspicious acetowhite tissue. Characteristic features suggestive of the degree of dysplasia have been described. ${ }^{26}$

-Figs. 1 to 4 represent the typical appearance under HRA of normal mucosa and various other findings.

The first reports in the literature of colposcopy being utilized for anorectal disease were submitted by O'Connor between 1977 and 1979. ${ }^{27-29}$ By 1979, he had compiled a

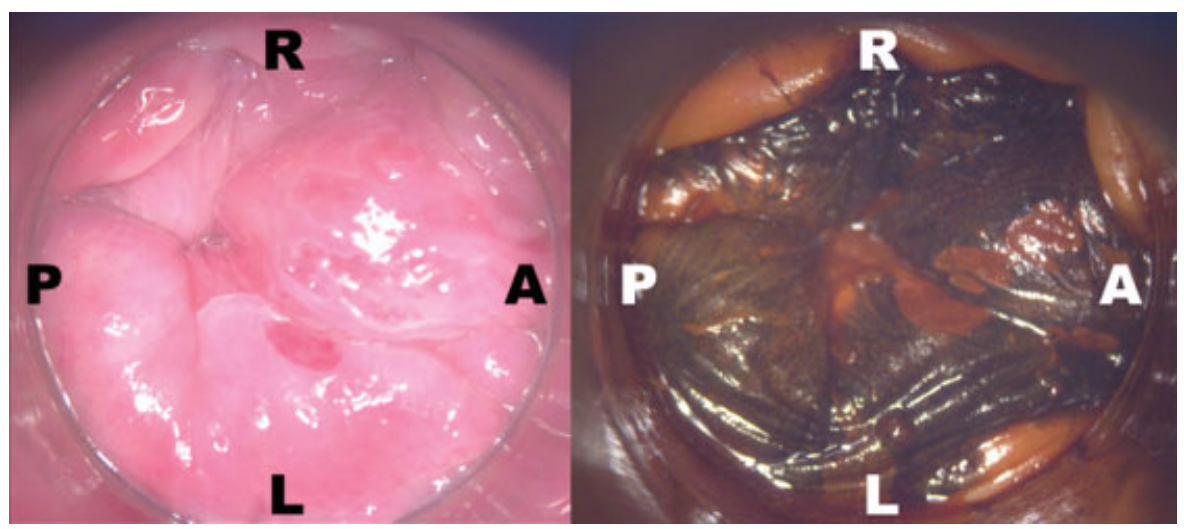

Fig. 1 Normal exam. Left image: Acetowhite exam. Right image: Lugol's iodine exam. (Left lateral position: L, left; R, right; A, anterior; P, posterior). 


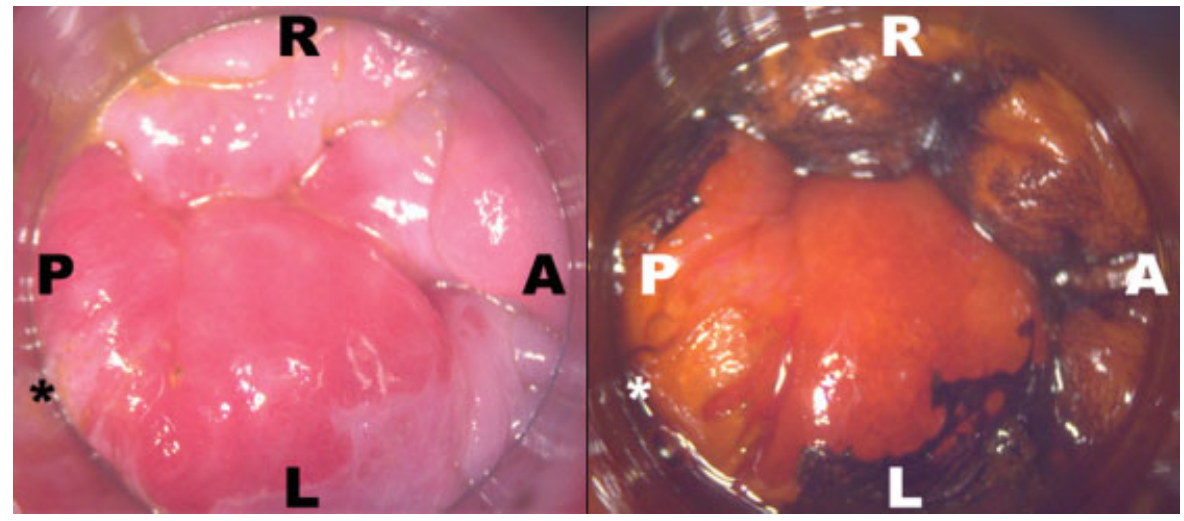

Fig. 2 LSIL/AIN I. Left image: Left posterior squamocolumnar junction enhancement and coarse punctation. Right image: Negative iodine uptake $\left.{ }^{*}\right)$. (Left lateral position: L, left; R, right; A, anterior; P, posterior).

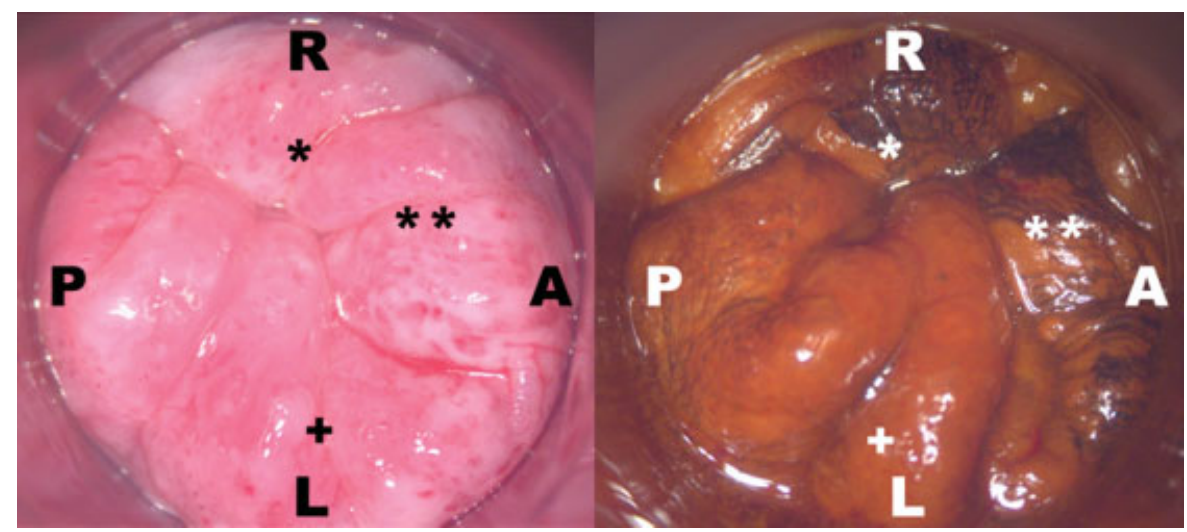

Fig. 3 HSIL/AIN 2-3. Left image: There are three findings. (1) Right lateral acetowhite enhancement of squamocolumnar junction (*). (2) Left Lateral acetowhite with coarse punctation $(+)$. (3) Right anterior acetowhite enhancement with coarse punctation $\left(^{* *}\right)$. Right image: (1) Lugol negative right lateral squamocolumnar junction finding $\left({ }^{*}\right)$. (2) Lugol negative left lateral finding $(+)$. (3) Lugol negative right anterior finding $\left({ }^{* *}\right)$. (Left lateral position: L, left; R, right; A, anterior; P, posterior).

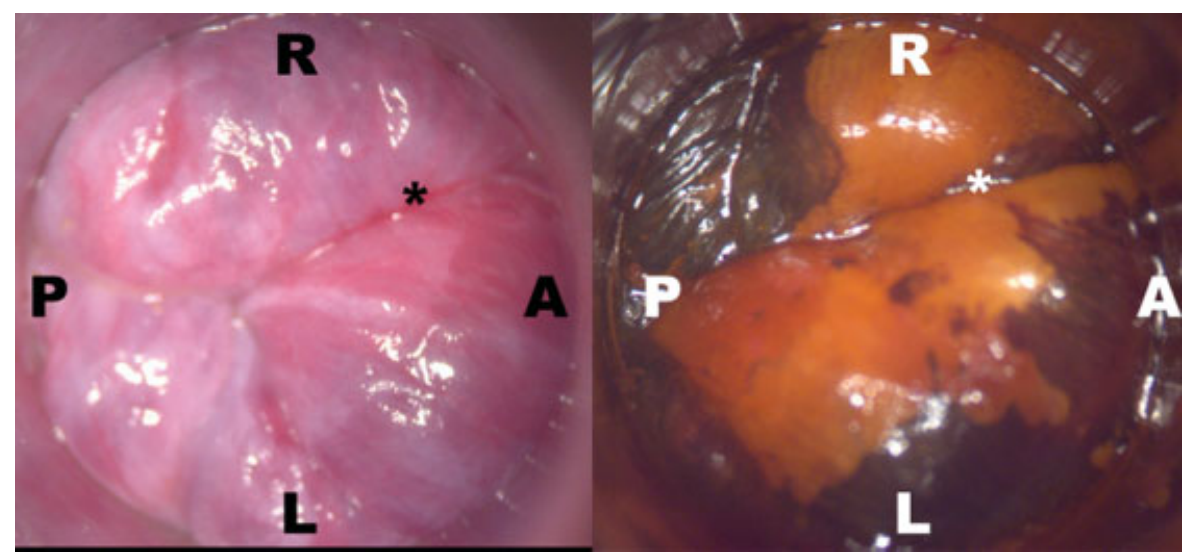

Fig. 4 AIN 3. Left image: Right anterior increased acetowhite enhancement $\left({ }^{*}\right)$. Right image: Right anterior acetowhite finding with negative Logol's uptake $\left(^{*}\right)$. (Left lateral position: L, left; R, right; A, anterior; P, posterior).

series of 500 patients who underwent colposcopy with the addition of cytologic staining with Lugol's iodine and acetic acid, and applied the standards for visualization of vulvar and cervical lesions based on alterations in color, contour, and blood vessel pattern. The indications for HRA were pruritus ani, ulcer, condyloma, mass lesion, occult bleeding, previous anorectal malignancy, and symptoms not explained by conventional anoscopy. He found 238 lesions in 500 patients, of which he characterized 98 as undetectable without colposcopy, or a miss rate of $41 \%$ for standard examination. These lesions represented a variety of entities such as carcinoids, one adenocarcinoma, and fistula. However, 80 lesions were condyloma (15), Bowen's disease (19), squamous cell carcinoma (2), or dysplasia (44). Although his 
series lacks any formal statistical analysis or patient demographics, $16 \%$ of patients ( 80 of 500 ) and $34 \%$ of lesions ( 80 of 238) were found to be HPV related.

\section{HPV Similarly Causes Anal Dysplasia and Anal Dysplasia Can Cause Anal Cancer}

It is currently established that HPV can cause anal dysplasia. It is also known that high-grade anal dysplasia can progress to anal cancer. There is a strong causal association between HPV and anal squamous cell carcinoma analogous to what has been established in cervical cancer. It has been shown that the same HPV types that infect the epithelium of the lower female genital tract can infect anal epithelium resulting in similar lesions. ${ }^{30,31}$ Furthermore, squamous cell carcinomas as well as high-grade dysplastic lesions of the cervix and anus are histologically similar. ${ }^{31}$

In the early 1960s, malignant changes, carcinoma in situ, and squamous cell carcinoma had been seen in anal warts. ${ }^{32}$ By the early 1970s, electron microscopy allowed for the isolation and visualization of papillomavirus particles from anal condyloma acuminata within an area of carcinoma in situ. ${ }^{33}$ These are among the first reports in the literature linking HPV to anal dysplasia and anal cancer. Numerous instigations followed, confirming the causal nature of HPV and anal dysplasia as well as the progression to cancer from high-grade dysplastic lesions of the anal canal. For example, Duggan and colleagues noted HPV DNA in 73\% of condylomas and also in $67 \%$ of AIN lesions. ${ }^{30}$

Frazer et al noted a high incidence of cytological changes characteristic of HPV infection and of dysplasia in the anorectal epithelium in men who have sex with men (MSM). ${ }^{34}$ They also described an association between anal dysplasia and frequent anoreceptive intercourse, HIV seropositivity, and low CD4/CD8 ratio. They applied the analogy of HPV-associated changes in the cervical epithelium leading to cervical dysplasia and carcinoma and concluded that dysplasia in the anorectal epithelium may be a marker for future anorectal carcinoma.

Further studies continue to validate this association. Devaraj and Cosman conducted a study in which men with HIV and anal dysplasia were observed with no treatment. ${ }^{35}$ Forty percent of their overall cohort of 98 HIV-positive MSM had anal dysplasia. Three of these men developed squamous cell carcinoma of the anus, describing a progression rate of $\sim 7.5 \%$ over 130 months, supporting the contention that high-grade anal dysplasia without treatment can progress to anal squamous cell carcinoma. Berry et $\mathrm{a}^{36}$ retrospectively demonstrated that anal carcinoma developed at previously biopsied sites of high-grade dysplasia in 27 out of 72 anal cancers studied (37.5\%), illustrating the potential for development of anal squamous cell carcinoma from highgrade anal dysplasia.

Additional studies have shown that HPV is not only associated with anal dysplasia but also that specific genotypes can be of higher risk for the development of high-grade dysplasia. High-grade intraepithelial lesions are associated with high-risk HPV genotypes, particularly HPV 16 and $18 .^{30,31,37}$ Furthermore, HIV-positive patients have a higher prevalence of anal dysplasia ${ }^{38}$ and high-risk genotypes. ${ }^{37}$
Although the natural history of HPV and anal dysplasia is not exactly understood, our current understanding is that there is a strong causal relationship between HPV and anal squamous cell carcinoma, especially within the HIV-positive MSM population. The parallels between cervical and anal cancer and HPV are not perfect, however. High-grade cervical dysplasia progresses to cervical cancer in $~ 30$ to $36 \%$ of cases at 20 to 30 years. ${ }^{20,35}$ Several reports suggest that essentially $100 \%$ of HIV-positive individuals develop high-grade anal dysplasia, ${ }^{20,39,40}$ with Chang and colleagues ${ }^{39}$ reporting this recurrence rate within 12 months of treatment of preexisting HSIL. The Centers for Disease Control and Prevention reported in 2016 that 1.2 million individuals are living with HIV in the United States. ${ }^{41}$ Assuming a $100 \%$ prevalence of high-grade anal dysplasia in this population, and with the 30 to $36 \%$ lifetime incidence reported in cervical cancer extrapolated to anal cancer, this would be equivalent to more than 10 times the reported incidence of anal cancer than occurs in the yearly. Multiple reports have cited a 1 to $3 \%$ rate of progression from HSIL to anal carcinoma with nearly all cases occurring in HIV-positive individuals. ${ }^{42-45}$ This would suggest that anal HSIL is a more prevalent yet more indolent process than cervical HSIL. ${ }^{35}$

\section{HRA Can Be Used to Manage Anal Dysplasia}

Screening and surveillance for anal SIL is clearly important for the early detection and prevention of squamous cell carcinoma of the anus. There are multiple methods by which both screening and surveillance can be completed, including anal cytology, digital rectal examination, standard anoscopy, and HRA. Of these methods, HRA has emerged as an effective way of detecting anal dysplasia especially in high-risk populations such as HIV-positive MSM, and short of histologic examination ${ }^{23}$ has been considered the gold standard to which other methods of detection are compared. ${ }^{46,47}$

Cytologic screening such as the anal Pap smear tends to underestimate the true level of histological dysplasia. ${ }^{37}$ This screening test is performed by blindly swabbing the anal transition zone. The cytology is then interpreted according to the Bethesda classification. ${ }^{20}$ Although in the past this was thought to be more useful, ${ }^{47}$ currently it is felt that in highrisk populations such as HIV-positive MSM, anal cytology can be inaccurate and has been shown to not correlate with histology. ${ }^{48,49}$

Jay and coworkers ${ }^{26}$ used cervical colposcopic methodology to establish that HSILs could be distinguished from lowgrade squamous intraepithelial lesions (LSILs) much like intraepithelial lesions described in the cervix. Using HRA with cytologic staining as described earlier, the anal canal and transition zone are visualized under high-resolution microscopy. Suspicious areas are then biopsied, or HRA-targeted therapy can be performed. ${ }^{50}$ Pineda et $\mathrm{al}^{51}$ showed that HRA in HIV-negative patients offered an effective method of controlling anal dysplasia in the immunocompetent patient with minimal morbidity, with a $2.4 \%$ rate of progression to cancer and a $45 \%$ recurrence rate of high-grade dysplasia. In a second longer term study including immunocompromised patients, Pineda et $\mathrm{al}^{42}$ concluded that HRA targeted surgical 
destruction combined with office-based surveillance and directed therapy is effective in controlling HSIL and is superior to reports of expectant management or traditional mapping procedures. The recurrence rate was $57 \%$ at 19 months and progression rate was $1.2 \%$ with complications in $4 \%$. At last visit, $78 \%$ of patients had no high-grade intraepithelial lesions.

\section{Those at Highest Risk for Anal Dysplasia and Anal Cancer Were Identified as Men Who Practice Anoreceptive Intercourse with Men Who Are HIV Positive}

Early reports like the one by Daling et $\mathrm{al}^{52}$ identified condyloma as conferring a 20-fold risk for anal cancer over those without condyloma. They also noted that anoreceptive intercourse conferred a 25-fold risk for anal cancer. Prior to the HIV pandemic, numerous reports cited the incidence of anal cancer in MSM as similar or higher than rates for cervical cancer before the cervical cancer screening era (35 per $100,000)$ as compared with the general population of 2 to 3 per $100,000 .^{23,46,53}$ In this setting, it was found that HIV seropositive status is a risk factor for both anal dysplasia and anal cancer. ${ }^{34}$ Readers are directed to the epidemiology article of this volume for a detailed discussion of this relationship.

\section{HIV Positivity Is a Risk Factor for HPV Infection, Dysplasia, and Anal Cancer}

\section{HRA Has Been Used Most Often to Detect SIL in HIV+ MSM}

The preponderance of reports in the literature has focused on the usage of HRA in the screening and management of anal dysplasia for HIV-positive MSM, and surveillance and treatment has also been targeted to this patient population. This has been due to the strong inferential data that have been discussed to this point identifying this population as currently being the one with the highest need, in terms of prevalence of dysplasia and risk of development of anal squamous carcinoma. Although no randomized controlled trials exist, precedents do exist in other analogous instances. For example, in screening for cervical cancer, no randomized data support the use of the Pap test, ${ }^{14}$ but its impact on the disease has been accepted worldwide and is formally incorporated into international screening guidelines. ${ }^{19}$ Similarly, no randomized data exist for the prevention of colorectal cancer by screening colonoscopy, but this has become widely accepted since the seminal report by Winawer and colleagues in $1993 .^{54}$

\section{Other Populations}

The literature is scarce at best in other populations. In women for example, a systematic review ${ }^{55}$ from 2015 identified 23 reports on the epidemiology of anal dysplasia and anal cancer in women as compared with hundreds of studies in HIV-positive MSM. They identified the prevalence of highrisk HPV as between 16 and 85\% in HIV-positive women, and between 4 and $85 \%$ in HIV-negative women. HPV-related pathology of the vulva, vagina, or cervix conferred an approximately fourfold risk for anal HPV as compared with women with no HPV-related history. HSIL was found in 3 to $26 \%$ of HIV-positive women as compared with 0 to $3 \%$ of women with no known elevated risk factors (HIV positive or lower genital tract pathology). The prevalence of SIL was found to be $50 \%$ in HIV-positive women in one cross-section of sub-Saharan Africa, ${ }^{56} 0 \%$ in a cohort from Thailand, ${ }^{57}$ and $7.5 \%$ in a group of women from India. ${ }^{58}$ The group from India ${ }^{58}$ did note that all women with anal dysplasia reported a history of anoreceptive intercourse. HRA was used to evaluate women with a history of lower genital tract pathology (high-risk group). ${ }^{59}$ A $13.4 \%$ prevalence of anal dysplasia was found in the high-risk group versus $0 \%$ in the low-risk group. Overall, the incidence of anal cancer appears to be lower in HIV-positive women than in HIV-positive MSM, but higher than the general population. However, it is possible that these rates are falsely low and subject to publication bias, in that most studies on women date from the late 2000s and even 2010s, where studies on MSM date back to the early 1980s. Further, it should be noted that nearly all studies are essentially extended case reports, with small numbers and mostly descriptive statistics versus any formal analysis of the effectiveness of any particular treatment or screening regimen. That said, higher prevalences and clinically significant detection of anal dysplasia in high-risk women (generally HIV positive and history of lower genital tract pathology) speak to the utility of HRA in this population.

Transplant patients are another population at elevated risk for anal cancer due to immunosuppression. Despite this, Ogilvie and colleagues ${ }^{60}$ noted insufficient evidence to support any formalized screening program in transplant patients. Thus, they undertook HRA and cytology in a small cohort of renal transplant patients at the University of Minnesota. They found a $6 \%$ rate of anal dysplasia by HRA and biopsy. They also noted poor ability of cytology to detect abnormalities (sensitivity $17 \%$ ). Their report also suffers from low study numbers.

In light of the paucity of data and currently understood lower rates of anal dysplasia and anal cancer in other populations, Gosens et $\mathrm{al}^{46}$ discussed HPV, anal cancer, and HRA within the classic criteria for an effective screening program for any disease entity. These include, among others, a disease that is an important health problem, a recognizable latent stage, suitable and acceptable testing, and an agreed upon treatment population. Given the high prevalence of HPV, highrisk HPV subtypes, HSIL, and higher risk of anal cancer over any population studied to date, they identified HIV-positive MSM as the target population for screening programs.

\section{How Is HRA Used Today and How Well Does HRA Work to Detect SIL in HIV+ MSM?}

Data on performance characteristics of HRA for the detection of anal dysplasia are difficult to interpret. In comparative studies, HRA was considered the standard by which cytology was measured. Most reports sought to clarify the performance of cytology as an initial screening tool, for which HRA was performed in the setting of an abnormal or equivocal test. There is significant heterogeneity in study design, in which 
some studies did not seek to match cytology and HRA on patient, ${ }^{48,61,62}$ while other authors performed cytology and HRA on all patients. ${ }^{49,63-66}$ HIV positivity, MSM, and gender of included patients were not consistent across studies.

Taken as a whole, there appears to be poor correlation between HRA and cytology. Sensitivity of cytology was highly variable, ranging from 16 to $98 \%$, while specificity ranged from 38 to $90 \% .{ }^{48,49,61,65,66}$ Dietrich et al ${ }^{63}$ reported a high correlation between cytology and HRA (67\%) but noted that $71 \%$ of atypical cells of unknown significance (ASCUS) harbored highgrade dysplasia on HRA, while Cranston et $\mathrm{al}^{62}$ reported nearly half of all ASCUS or LSIL found on cytology led to HSIL on HRA. Clinically, the specter of cytologic inaccuracy is managed by lowering the threshold to move to HRA at any suggestion of dysplasia. ${ }^{64}$ These data would suggest and support the contention that HRA is currently the standard for survey of the anal canal for dysplasia.

Although comparative data suggest that HRA is the superior modality as compared with cytology, the accuracy of HRA itself has been poorly studied. Richel et $\mathrm{al}^{53}$ investigated the interobserver agreement for HRA specimens and found good correlation for condylomatous lesions (kappa coefficient of 0.65 ) and poor to moderate correlation for flat lesions (kappa coefficient of $0.08-0.54$ ). The highest positive predictive value of $40 \%$ was found for the combination of punctation, flat leukoplakia, and atypical vessel pattern. In 2004, Mathews et $\mathrm{al}^{64}$ found lower agreement with a kappa coefficient of 0.32 (poor) for visual HRA findings compared with histopathological findings on biopsy. Three years later in 2007, Mathews et $\mathrm{al}^{67}$ reported a nearly identical kappa coefficient (0.29) for correlation between HRA and histopathology, and also noted a strong relationship between operator experience and correlation. The only other study directly comparing colposcopic findings with histopathology is the oft-cited study by Jay and colleagues ${ }^{26}$ which applied cervical colposcopic visual standards to HRA. The sensitivities for different findings ranged from 12 to $84 \%$, while positive predictive values ranged from 40 to $61 \%$.

The goal of any screening program is to prevent the endpoint disease; in this case, screening for anal dysplasia to prevent anal cancer. Multiple reports suggest a 1 to $3 \%$ incidence of anal cancer in patients with HSIL, with nearly all cases occurring in HIV-positive individuals. ${ }^{42-45}$ Mathews et $\mathrm{al}^{67}$ also noted that there is an important distinction between preventing precursor lesions that can develop into anal cancer and preventing anal cancer. Very few reports are available that directly address this topic. In 2015, Crawshaw and colleagues ${ }^{45}$ compared two groups of patients, all with biopsy-proven anal dysplasia but not anal cancer. Both groups had a similar proportion of HIV-positive individuals of around 75\%. One group (expectant management) underwent routine screening with cytology, digital rectal exam, anoscopy, and either ablation or imiquimod treatment of clinically evident lesions. The other group (HRA) underwent cytology then HRA at routine intervals and treatment of suspicious lesions. After a 3-year follow-up period, two patients in the expectant management group and one in the HRA group progressed to carcinoma. The 5-year progression rate to cancer was $6 \%$ in the expectant management group and $4.5 \%$ in the HRA group which was not statistically significant $(p=0.37)$. Mathews et $\mathrm{al}^{67}$ studied a cohort of 5,000 patients pre- and postimplementation of an HRA screening program and found similar incidences of anal cancer pre- and post-HRA (11 vs. 17 cases) and nearly identical numbers of anal cancer in those screened versus not screened after the program was implemented ( 8 vs. 9, $p=1.0)$.

On a population level, the preceding controversies undoubtedly underlie the lack of consistent, standardized guidelines on a national or global level. The 2015 Centers for Disease Control and Prevention guidelines ${ }^{68}$ concluded that data are insufficient to recommend any screening for anal cancer in any individuals with HIV infection, HIV-positive MSM, or the general population. They do acknowledge that some centers perform HRA for abnormal cytology but make no further recommendation. The U.S. Preventative Task Force has no topics on anal cancer. ${ }^{69}$ The most recent (2011) guidelines of the International Union Against Sexually Transmitted Infections also makes no mention of HRA or any screening modality for anal dysplasia for the European, AsiaPacific, or Australian region. ${ }^{70}$

Currently in the United States, the two most robust sets of guidelines come from New York and California. The National Aids Treatment Advocacy Project (NATAP) ${ }^{71}$ outlines the New York state guidelines which pertain only to HIV-positive individuals:

- History, external exam, and digital rectal exam.

- Anal cytology yearly for HIV-positive MSM, history of anogenital condyloma, or women with abnormal cervical and/or vulvar histology.

- HRA and biopsy for any patient with abnormal cytology.

The University of California, San Francisco algorithm is as follows ${ }^{62}$ :

- Yearly cytology in HIV-positive individuals and every 2 to 3 years in the HIV-negative population.

- HRA for any abnormal cytology.

- HRA every 6 months for LSIL.

- Treatment for HSIL.

It is widely accepted that HRA is difficult to learn and has a steep learning curve, which in large part contributes to a shortage of skilled providers who perform HRA. ${ }^{23,49,53,72,73}$ Furthermore, the procedure itself is not standardized, variations in technique are poorly documented, and the training backgrounds of practitioners vary widely. ${ }^{73}$ As a result, the International Anal Neoplasia Society set forth guidelines for practice standards in the detection of anal cancer precursors in $2016 .^{73}$ In addition to basic equipment and logistic requirements, they outline basic practice competencies, training standards, and minimum documentation requirements. Furthermore, a minimum of 50 HRA procedures per year is suggested, with 20 diagnoses of HSIL, greater than $90 \%$ rate of visualization of the squamocolumnar junction, distal anal canal and perianal margin, and greater than $90 \%$ rate of procedural duration of more than 5 minutes but less than 15 minutes. 
Finally, any ablative therapy directed at anal dysplasia can be utilized via HRA. These would include electrocautery, ${ }^{45}$ local immunomodulators such as imiquimod, ${ }^{45}$ acetic acid, ${ }^{45}$ infrared coagulation, ${ }^{44}$ and excisional biopsy. ${ }^{39}$ Although as previously noted, recurrence rates are high, with one report noting an $80 \%$ recurrence after the first treatment, and an identical recurrence after the second treatment. ${ }^{44}$

\section{How Will HRA Be Used in the Future?}

The future of HRA will likely be fueled by today's deficiencies in the understanding, screening, and managing anal dysplasia and anal cancer.

More study is needed on the natural history of anal dysplasia in the general population, women, and relatively unstudied populations such as transplant patients. Improved screening methods providing better detection and also correlation with degree of dysplasia are in critical need. Access to HRA is another barrier to screening and treatment. Practice standard guidelines like the ones set forth by Hillman and colleagues $^{73}$ are an important first step in promulgating HRA. Improved local therapies associated with decreased rates of recurrence will help HRA assume more of a role in treatment versus primary screening. Greater awareness and less stigma as the incidence and prevalence of anal dysplasia and anal cancer continue to rise is critical on a population level. Increased awareness of high-risk populations and of options for screening and treatment is also important from a health care provider's perspective. Readers are directed elsewhere in this volume for a discussion of the impact of multidisciplinary awareness in improving care for high-risk patients with SIL.

\section{Photo Credits}

Ami Multani, MD, Primary Care, Fenway Community Health Center, Division of Infectious Diseases, Beth Israel Deaconess Medical Center, Instructor in Medicine, Harvard Medical School.

\section{Acknowledgments}

The authors wish to thank P. Alex Leahey, MD, of Kaiser Permanente Northwest Medical Group in Portland, Oregon, for his assistance in obtaining images for this chapter.

\section{References}

1 Rotkin ID. A comparison review of key epidemiological studies in cervical cancer related to current searches for transmissible agents. Cancer Res 1973;33(06):1353-1367

2 Rowson KEK, Mahy BW. Human papova (wart) virus. Bacteriol Rev 1967;31(02):110-131

3 Almeida JD, Oriel JD, Stannard LM. Characterization of the virus found in human genital warts. Microbios 1969;1:225-232

4 Laverty CR, Russell P, Hills E, Booth N. The significance of noncondylomatous wart virus infection of the cervical transformation zone. A review with discussion of two illustrative cases. Acta Cytol 1978;22(04):195-201

5 Laverty CR, Booth N, Hills E, Cossart Y, Wills EJ. Noncondylomatous wart virus infection of the postmenopausal cervix. Pathology $1978 ; 10(04): 373-378$
6 Reid R, Laverty CR, Coppleson M, Isarangkul W, Hills E. Noncondylomatous cervical wart virus infection. Obstet Gynecol 1980;55 (04):476-483

7 Reid R, Stanhope CR, Herschman BR, Booth E, Phibbs GD, Smith JP. Genital warts and cervical cancer. I. Evidence of an association between subclinical papillomavirus infection and cervical malignancy. Cancer 1982;50(02):377-387

8 Reid R. Genital warts and cervical cancer. II. Is human papillomavirus infection the trigger to cervical carcinogenesis? Gynecol Oncol 1983;15(02):239-252

9 Reid R, Crum CP, Herschman BR, et al. Genital warts and cervical cancer. III. Subclinical papillomaviral infection and cervical neoplasia are linked by a spectrum of continuous morphologic and biologic change. Cancer 1984;53(04):943-953

10 Pilotti S, Rilke F, Alasio L, Fontanelli R. Histologic evidence for an association of cervical intraepithelial neoplasia with human papilloma virus infection. Diagn Gynecol Obstet 1982;4(04): 357-362

11 Boshart M, Gissmann L, Ikenberg H, Kleinheinz A, Scheurlen W, zur Hausen $H$. A new type of papillomavirus DNA, its presence in genital cancer biopsies and in cell lines derived from cervical cancer. EMBO J 1984;3(05):1151-1157

12 Ho GYF, Bierman R, Beardsley L, Chang CJ, Burk RD. Natural history of cervicovaginal papillomavirus infection in young women. $\mathrm{N}$ Engl J Med 1998;338(07):423-428

13 Fusco E, Padula F, Mancini E, Cavaliere A, Grubisic G. History of colposcopy: a brief biography of Hinselmann. J Prenat Med 2008;2 (02):19-23

14 Lees BF, Erickson BK, Huh WK. Cervical cancer screening: evidence behind the guidelines. Am J Obstet Gynecol 2016;214(04):438-443

15 Apgar BS, Brotzman GL, Spitzer M. Colposcopy: Principles and Practice. Elsevier Health Sciences; 2008

16 Coppleson M. Colposcopic features of papillomaviral infection and premalignancy in the female lower genital tract. Obstet Gynecol Clin North Am 1987;14(02):471-494

17 Krumholz BA, Knapp RC. Colposcopic selection of biopsy sites. Obstet Gynecol 1972;39(01):22-26

18 Burke L, Antonioli D, Knapp RC, Friedman EA. Vaginal adenosis. Correlation of colposcopic and pathologic findings. Obstet Gynecol 1974;44(02):257-264

19 Massad LS, Einstein MH, Huh WK, et al; 2012 ASCCP Consensus Guidelines Conference. 2012 updated consensus guidelines for the management of abnormal cervical cancer screening tests and cancer precursors. J Low Genit Tract Dis 2013;17 (5, Suppl 1):S1-S27

20 Lundberg GD; National Cancer Institute Workshop. The 1988 Bethesda System for reporting cervical/vaginal cytological diagnoses. JAMA 1989;262(07):931-934

21 Schlichte MJ, Guidry J. Current cervical carcinoma screening guidelines. J Clin Med 2015;4(05):918-932

22 American Society for Colposcopy and Cervical Pathology. (August 2014). Updated Consensus Guidelines for Managing Abnormal Cervical Cancer Screening Tests and Cancer Precursors. Available at: http://www.asccp.org/asccp-guidelines

23 Darragh TM, Winkler B. Anal cancer and cervical cancer screening: key differences. Cancer Cytopathol 2011;119(01):5-19

24 Palefsky JM. Practising high-resolution anoscopy. Sex Health 2012;9(06):580-586

25 Albuquerque A. High-resolution anoscopy: unchartered territory for gastroenterologists? World J Gastrointest Endosc 2015;7(13): 1083-1087

26 Jay N, Berry JM, Hogeboom CJ, Holly EA, Darragh TM, Palefsky JM. Colposcopic appearance of anal squamous intraepithelial lesions: relationship to histopathology. Dis Colon Rectum 1997;40(08): 919-928

27 O'Connor JJ. The study of anorectal disease by colposcopy. Dis Colon Rectum 1977;20(07):570-572 
28 O'Connor JJ. Colposcopy in anorectal disease. Surg Gynecol Obstet 1978;147(06):924-925

29 O'Connor JJ. Colposcopy in anorectal disease. South Med J 1979;72 (12):1559-1560

30 Duggan MA, Boras VF, Inoue M, McGregor SE, Robertson DI. Human papillomavirus DNA determination of anal condylomata, dysplasias, and squamous carcinomas with in situ hybridization. Am J Clin Pathol 1989;92(01):16-21

31 Hoots BE, Palefsky JM, Pimenta JM, Smith JS. Human papillomavirus type distribution in anal cancer and anal intraepithelial lesions. Int J Cancer 2009;124(10):2375-2383

32 Siegel A. Malignant transformation of condyloma acuminatum. Review of the literature and report of a case. Am J Surg 1962; 103:613-617

33 Oriel JD, Almeida JD. Demonstration of virus particles in human genital warts. Br J Vener Dis 1970;46(01):37-42

34 Frazer IH, Medley G, Crapper RM, Brown TC, Mackay IR. Association between anorectal dysplasia, human papillomavirus, and human immunodeficiency virus infection in homosexual men. Lancet 1986;2(8508):657-660

35 Devaraj B, Cosman BC. Expectant management of anal squamous dysplasia in patients with HIV. Dis Colon Rectum 2006;49(01): 36-40

36 Berry JM, Jay N, Cranston RD, et al. Progression of anal high-grade squamous intraepithelial lesions to invasive anal cancer among HIV-infected men who have sex with men. Int J Cancer 2014;134 (05):1147-1155

37 Sendagorta E, Herranz P, Guadalajara H, et al. Prevalence of abnormal anal cytology and high-grade squamous intraepithelial lesions among a cohort of HIV-infected men who have sex with men. Dis Colon Rectum 2014;57(04):475-481

38 Palefsky JM. Anal squamous intraepithelial lesions in human immunodeficiency virus-positive men and women. Semin Oncol 2000;27(04):471-479

39 Chang GJ, Berry JM, Jay N, Palefsky JM, Welton ML. Surgical treatment of high-grade anal squamous intraepithelial lesions: a prospective study. Dis Colon Rectum 2002;45(04):453-458

40 Ontario HQ; Health Quality Ontario. Anal dysplasia screening: an evidence-based analysis. Ont Health Technol Assess Ser 2007;7 (04):1-43

41 Centers for Disease Control and Prevention. (December 2016). HIV in the United States: At a Glance. Available at: https://www. cdc.gov/hiv/statistics/overview/ataglance.html

42 Pineda CE, Berry JM, Jay N, Palefsky JM, Welton ML. High-resolution anoscopy targeted surgical destruction of anal high-grade squamous intraepithelial lesions: a ten-year experience. Dis Colon Rectum 2008;51(06):829-835, discussion 835-837

43 Dalla Pria A, Alfa-Wali M, Fox P, et al. High-resolution anoscopy screening of HIV-positive MSM: longitudinal results from a pilot study. AIDS 2014;28(06):861-867

44 Goldstone SE, Johnstone AA, Moshier EL. Long-term outcome of ablation of anal high-grade squamous intraepithelial lesions: recurrence and incidence of cancer. Dis Colon Rectum 2014;57 (03):316-323

45 Crawshaw BP, Russ AJ, Stein SL, et al. High-resolution anoscopy or expectant management for anal intraepithelial neoplasia for the prevention of anal cancer: is there really a difference? Dis Colon Rectum 2015;58(01):53-59

46 Gosens KCM, Richel O, Prins JM. Human papillomavirus as a cause of anal cancer and the role of screening. Curr Opin Infect Dis 2017; 30(01):87-92

47 Pineda CE, Welton ML. Management of anal squamous intraepithelial lesions. Clin Colon Rectal Surg 2009;22(02):94-101

48 Panther LA, Wagner K, Proper J, et al. High resolution anoscopy findings for men who have sex with men: inaccuracy of anal cytology as a predictor of histologic high-grade anal intraepithelial neoplasia and the impact of HIV serostatus. Clin Infect Dis 2004;38(10):1490-1492
49 Berry JM, Palefsky JM, Jay N, Cheng SC, Darragh TM, Chin-Hong PV. Performance characteristics of anal cytology and human papillomavirus testing in patients with high-resolution anoscopyguided biopsy of high-grade anal intraepithelial neoplasia. Dis Colon Rectum 2009;52(02):239-247

50 Berry JM, Jay N, Palefsky JM, Welton M. State-of-the-Art of high resolution anoscopy as a tool to manage patients at risk of anal cancer. Semin Colon Rectal Surg 2004;15(04):218-226

51 Pineda CE, Berry JM, Jay N, Palefsky JM, Welton ML. High resolution anoscopy in the planned staged treatment of anal squamous intraepithelial lesions in HIV-negative patients. J Gastrointest Surg 2007;11(11):1410-1415, discussion 14151416

52 Daling JR, Weiss NS, Hislop TG, et al. Sexual practices, sexually transmitted diseases, and the incidence of anal cancer. N Engl J Med 1987;317(16):973-977

53 Richel O, Hallensleben NDL, Kreuter A, van Noesel CJ, Prins JM, de Vries HJ. High-resolution anoscopy: clinical features of anal intraepithelial neoplasia in HIV-positive men. Dis Colon Rectum 2013;56(11):1237-1242

54 Winawer SJ, Zauber AG, Ho MN, et al; The National Polyp Study Workgroup. Prevention of colorectal cancer by colonoscopic polypectomy. N Engl J Med 1993;329(27):1977-1981

55 Stier EA, Sebring MC, Mendez AE, Ba FS, Trimble DD, Chiao EY. Prevalence of anal human papillomavirus infection and anal HPVrelated disorders in women: a systematic review. Am J Obstet Gynecol 2015;213(03):278-309

56 Goeieman BJ, Firnhaber CS, Jong E, et al. Prevalence of anal HPV and anal dysplasia in HIV-infected women from Johannesburg, South Africa. J Acquir Immune Defic Syndr 2017;75(03):e59-e64

57 Ramautarsing RA, Phanuphak N, Chaithongwongwatthana S, et al. Cervical and anal HPV infection: cytological and histological abnormalities in HIV-infected women in Thailand. J Virus Erad 2015;1(02):96-102

58 Bisherwal K, Pandhi D, Singal A, Guleria K, Mishra K. Evaluation of cervical and anal intraepithelial neoplasia in women attending a sexually transmitted infection clinic. Indian J Dermatol Venereol Leprol 2016;82(05):498-504

59 Robison K, Cronin B, Bregar A, et al. Anal cytology and human papillomavirus genotyping in women with a history of lower genital tract neoplasia compared with low-risk women. Obstet Gynecol 2015;126(06):1294-1300

60 Ogilvie JW Jr, Park IU, Downs LS, Anderson KE, Hansberger J, Madoff RD. Anal dysplasia in kidney transplant recipients. J Am Coll Surg 2008;207(06):914-921

61 Arain S, Walts AE, Thomas P, Bose S. The anal Pap smear: cytomorphology of squamous intraepithelial lesions. Cytojournal 2005;2(01):4-10

62 Cranston RD, Hart SD, Gornbein JA, Hirschowitz SL, Cortina G, Moe AA. The prevalence, and predictive value, of abnormal anal cytology to diagnose anal dysplasia in a population of HIVpositive men who have sex with men. Int J STD AIDS 2007;18 (02):77-80

63 Dietrich A, Hermans C, Heppt MV, Ruzicka T, Schauber J, Reinholz M. Human papillomavirus status, anal cytology and histopathological outcome in HIV-positive patients. J Eur Acad Dermatol Venereol 2015;29(10):2011-2018

64 Mathews WC, Sitapati A, Caperna JC, Barber RE, Tugend A, Go U. Measurement characteristics of anal cytology, histopathology, and high-resolution anoscopic visual impression in an anal dysplasia screening program. J Acquir Immune Defic Syndr 2004;37(05):1610-1615

65 Nahas CSR, da Silva Filho EV, Segurado AA, et al. Screening anal dysplasia in HIV-infected patients: is there an agreement between anal pap smear and high-resolution anoscopy-guided biopsy? Dis Colon Rectum 2009;52(11):1854-1860

66 Fox PA, Seet JE, Stebbing J, et al. The value of anal cytology and human papillomavirus typing in the detection of anal 
intraepithelial neoplasia: a review of cases from an anoscopy clinic. Sex Transm Infect 2005;81(02):142-146

67 Mathews C, Caperna J, Cachay ER, Cosman B. Early impact and performance characteristics of an established anal dysplasia screening program: program evaluation considerations. Open AIDS J 2007; 1:11-20

68 Centers for Disease Control and Prevention (May 2016). 2015 Sexually Transmitted Diseases Treatment Guidelines. HPV-associated Cancers and Precancers. Available at: https://www.cdc. gov/std/tg2015/hpv-cancer.htm

69 US Preventive Services Task Force. Recommendations. Available at: https://www.uspreventiveservicestaskforce.org/Page/Name/ recommendations
70 International Union Against Sexually Transmitted Infections. (September 2016). Guidelines. Available at: http://www.iusti. org/sti-information/guidelines/default.htm

71 National AIDS Treatment Advocacy Project. (July 2007). NYS Guidelines Recommendations on Anal Pap Smears. Available at: http:// www.natap.org/2010/HIV/032510_01.htm

72 Smyczek P, Singh AE, Romanowski B. Anal intraepithelial neoplasia: review and recommendations for screening and management. Int J STD AIDS 2013;24(11):843-851

73 Hillman RJ, Cuming T, Darragh T, et al. 2016 IANS International Guidelines for practice standards in the detection of anal cancer precursors. J Low Genit Tract Dis 2016;20(04): 283-291 